\title{
The Application of Lean Production Control Methods within a Process-Type Industry: The Case of Hydro Automotive Structures
}

\author{
Daryl Powell ${ }^{1}$, Erlend Alfnes ${ }^{1}$, and Marco Semini ${ }^{2}$ \\ ${ }^{1}$ Department of Production and Quality Engineering, Norwegian University of Science and \\ Technology, S.P. Andersens veg 5, NO-7465 Trondheim, Norway \\ ${ }^{2}$ Department of Industrial Management, SINTEF Technology and society, \\ S.P. Andersens veg 5, NO-7465 Trondheim, Norway
}

\begin{abstract}
Lean production has lead to substantial improvements in performance across many industries and is widely implemented today. Certain aspects of lean such as the focus on workplace organisation (5S) and total productive maintenance (TPM) have been applied to all types of industrial processes, while lean production control methods have mostly been applied in discrete and repetitive, assembly-type production. We believe that the real benefits of lean, for example throughput time and inventory reduction, are only realised when lean production control methods are implemented effectively. Therefore, we investigate the traditional lean production control methods of Heijunka and Kanban, and evaluate the concept of every product every (EPE) as an alternative lean production control method for the process-type industries.
\end{abstract}

Keywords: Process Industry, Lean, EPE, Cyclic Planning.

\section{Introduction}

In line with King (2009), we choose to categorise manufacturing environments into two distinct groups, assembly operations and process-type industries. Success in lean manufacturing has been largely associated with the automotive industry, typically in assembly operations, and has demonstrated improvements in quality, cost and delivery metrics. However, King (2009) suggests that there is a noticeable lack in lean adoption within the process-type industries. Although certain aspects of lean such as workplace organisation (5S) and Total Productive Maintenance (TPM) have been applied to all types of manufacturing environment, lean production control methods such as Heijunka and Kanban have mostly been applied in discrete, repetitive, assembly-type production. This signifies that Heijunka and Kanban are more appropriate for high volume, repetitive production of a low variety of highly standardized products. It is our opinion that the real benefits of lean production, for example throughput time and inventory reduction, are only truly realised when lean production control methods are implemented effectively. Therefore, the purpose of this paper is to demonstrate how to apply lean production control methods to those manufacturing environments less suited to Heijunka and Kanban, i.e. process-type industries. This research focuses on the application of lean production control methods in process-type industries, and 
is based on collaboration between two research projects funded by the Research Council of Norway, SFI Norman and CRASH. We would like to state that, although several other control methods exist, this paper only addresses those methods directly associated with lean production.

In this paper, we explore the concept of every product every (EPE) in order to introduce lean production control methods in process-type industries. The paper is structured as follows: We first classify manufacturing environments in line with King (2009). Then, we review traditional lean production control methods, which have been mainly applied to repetitive and discrete, assembly-type production. Next, we explore the concept of every product every (EPE). Finally, we demonstrate the application of EPE in a process-type industry through the use of a case study.

\section{Assembly Operations vs. Process-Type Industries}

Several authors attempt to categorize manufacturing activities (e.g. Hayes and Wheelwright (1979), Wild (1980), Hill (2005), amongst others). For the purpose of this paper, we have chosen a simplified categorization in line with King (2009), who proposes that manufacturing environments can be categorized into two groups: assembly operations and process industries. Many process industries operate in batch environments with likeness to discrete parts manufacture, whilst, on the other hand, many discrete parts manufacturers share characteristics with the process industries, where high volumes and large inflexible machines with long setup and changeover times require a high level of asset utilization.

Table 1 illustrates the distinguishing characteristics that separate the "process-type industries" from "assembly operations":

Table 1. Distinguishing Characteristics of Process-type Industries (adapted from King, 2009)

\begin{tabular}{|c|c|c|}
\hline & Assembly Operations & Process-type Industries \\
\hline Volume vs. Variety & High Volume, often Low Variety & High Volume, often High Variety \\
\hline $\begin{array}{c}\text { Throughput } \\
\text { Limited by: }\end{array}$ & Labour & Equipment \\
\hline $\begin{array}{c}\text { Size of Equipment } \\
\text { Cost of Stopping } \\
\text { and Restarting }\end{array}$ & Small, Simple, Easy-to-Move & Large, General Purpose, Complex \\
\hline $\begin{array}{c}\text { Product } \\
\text { Changeovers }\end{array}$ & Relatively Low & Relatively High \\
\hline $\begin{array}{c}\text { Material Flow } \\
\text { Pattern }\end{array}$ & Convergent, "A" Type & $\begin{array}{c}\text { Relatively Complex, Lost Time, Labour } \\
\text { AND Process Materials }\end{array}$ \\
\hline
\end{tabular}

From these characteristics, it is clear that it is not just the continuous process industries (e.g. oil refineries, chemicals, pulp and paper) that can be classed as process-type industries. Many discrete part manufactures also share these characteristics. It should also be noted that in a typical process industry, although there may be some degree of continuous processing, more often than not much of the production will still be performed in batches. 


\section{Lean Production Control Methods}

Lean production is to a large extent based on the manufacturing principles and work processes developed by Toyota in the 1940s. Lean is often defined in terms of waste reduction, hence a major aspect of the lean movement is just-in-time (JIT). JIT entails producing products in exactly the required quantity - just when they are needed, and not before (Shingo, 1989). Although some may consider other control methods within lean, for example two-bin and ConWIP, the traditional lean production control methods considered here are Heijunka and Kanban.

Heijunka is the method used in lean to level production in terms of both product volumes and product mix. Level production is a way of scheduling daily production for different types of products in a sequence to even out peaks and troughs in the quantities produced. Put simply, Bicheno and Holweg (2009) suggest that Heijunka is a post box system for Kanban cards that authorizes production in pitch incrementsized time slots. A typical pitch increment is between 10 and 30 minutes.

Kanban is a method of control designed to maximise the potential of the Toyota production system (Shingo, 1989). Kanban is typically the card that authorizes production of a certain product. When a product has been consumed from the finished goods inventory (or supermarket), a Kanban card is passed upstream (placed in the Heijunka box) to allow for replenishment of the product. Shingo (1989) suggests that Kanban can be applied only in plants involved in repetitive production, i.e. assembly operations. The application of Kanban is considered unsuitable in process-type industries. This is because, in process-type industries, large investments are made in even larger machines, often at the cost of substantial changeover times. With such large changeover times, introducing Kanban would have detrimental effects to the responsiveness of the production system, and may drastically increase production lead-times.

From previous experience, we recognise that although many of the lean tools and techniques have been applied successfully in all types of industrial processes, lean production control methods have rarely been applied to process-type industries, due to the characteristics defined in chapter three.

\section{Every Product Every (EPE): A Promising Concept for Process-Type Industries}

In the previous chapter, we explained that Heijunka and Kanban are difficult to apply in process-type industries due to the capacities of the production system, where resource utilisation is the key and large batch runs are the answer. To overcome this challenge and make lean flow possible in process-type industries, one approach is to develop a level production schedule, producing every product every cycle, which is also in line with lean production control principles. We suggest that levelled production is possible through the implementation of a fixed cyclic plan known as EPE. This plan coordinates production by assigning set periods of time to each product, in an optimum sequence. The optimum sequence is calculated by considering the changeover times between each of the products (Andersen et al., 1998).

The EPE concept stems as a result of the lean movement of the 1990s and is a descendent of cyclic planning. Rother and Shook (2003) identify EPE as one of several guidelines for creating a lean value stream. Inman and Jordan (1997) and Bicheno and 
Holweg (2009) also agree that producing every product every day is a key lean production principle. As EPE has not been properly defined in literature, we propose the following definition:

EPE is a lean production control method that involves creating a fixed cyclic plan through the levelling of product volume and mix, with a continuous focus on setup reduction.

Although in literature it may be unclear as to how EPE differs from Heijunka, we suggest that where EPE delivers a fixed cyclic production plan in terms of product sequence and volume, Heijunka assumes that changeover times are negligible, hence any sequence of products can be produced at any given time. In support of this, Bicheno and Holweg (2009) suggest that Heijunka be regarded as the final lean tool, because so much must be in place for its success, including setup reduction.

We suggest that the EPE concept is more applicable to process-type industries than Heijunka and Kanban as the length of an EPE cycle can be chosen for convenience, and may be a day, a week or longer. In fact, one cycle of an EPE plan could be considered as one column of a Heijunka box, as it is the time period over which the mixed-model quantity of each product is made.

Groenevelt et al. (1992) suggest that cyclic planning (such as EPE), is applicable in many environments and that the benefits range from shorter production leadtimes and lower work-in-process inventories and safety stocks, to improved material handling and material flow and increased customer responsiveness. We suggest that a fixed EPE plan will also deliver greater stability and predictability to the production environment, which results in less fire fighting and simplified coordination across the value-stream. Consequently, more time can be spent on improvement efforts, such as setup reduction.

The basis of EPE is to make each cycle of the plan as small as possible by doing as many changeovers as are feasible, in keeping with lean principles. Figure 1 demonstrates how production of every product every month can be developed into every product every day, assuming that enough capacity can be created by reducing changeover times or acquiring additional work centres:

One limitation of the fixed EPE plan, however, is its effect on a Make-to-Order (MTO) environment. If current setup times only allow for the production of every product every week, in MTO, this may in fact prolong the lead-time. Therefore, unless the capacity exists for the changeovers associated with every product every day, we would suggest that EPE is better suited to make-to-stock (MTS) environments. In addition, EPE may also be criticised for its requirement for a finished goods inventory, in order to deal with day-to-day fluctuations in demand. However, by

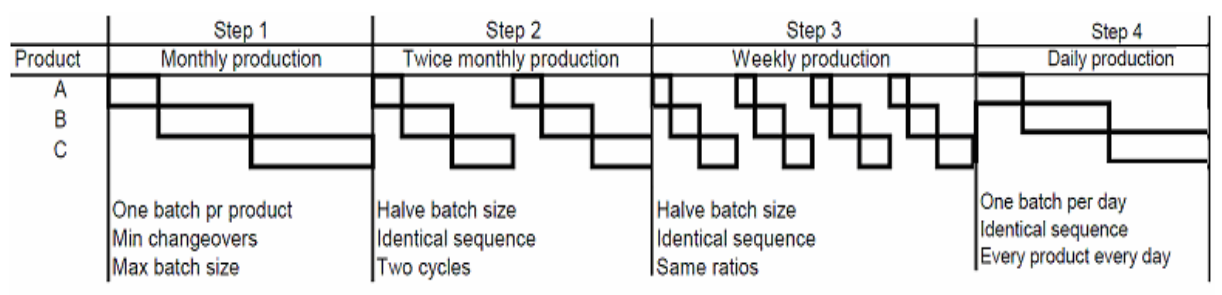

Fig. 1. Stages of development in EPE (adapted from Glenday and Sather, 2005) 
establishing stability thus reducing the need for fire fighting, the idea is to continuously work on setup reduction, allowing the length of the cycle to be continuously reduced, hence the finished goods inventory can also be reduced in size.

We suggest that three factors must be considered when designing an EPE plan, the first being runners, repeaters and strangers, which is a powerful idea for lean scheduling (Bicheno and Holweg, 2009). This involves dividing the products into those that have high volume, regular demand (runners), intermediate volume (repeaters) and low volume (strangers). Determining the optimum product sequence based on changeover times is the second factor. Finally, optimum batch size (EOQ vs. time available for setups) must also be considered. The design of an EPE plan is demonstrated in the following case study.

\section{The Case of Hydro Automotive Structures}

The characteristics that distinguish process-type industries, as described in Chapter three (particularly the divergent process structure, large equipment and complex changeovers), are representative of the situation at Hydro Automotive Structures Raufoss (HARA), in Norway. HARA supplies the automotive industry with crash management systems, developing and manufacturing bumper beams for almost all major original equipment manufacturers (OEMs).

This case study is taken from the previous work of Fauske et al. (2008) at SINTEF. In the case, the problems experienced by HARA can be defined as follows:

- Changes in demand and production breakdowns cause frequent plan changes and rush orders.

- Focus on high resource utilisation and large batch sizes results in subsequent high inventories.

- Poor information exchange between processes and lack of collaborative planning leads to confusion and delays in production.

Fauske et al. (2008) mapped the current material and information flows at HARA:

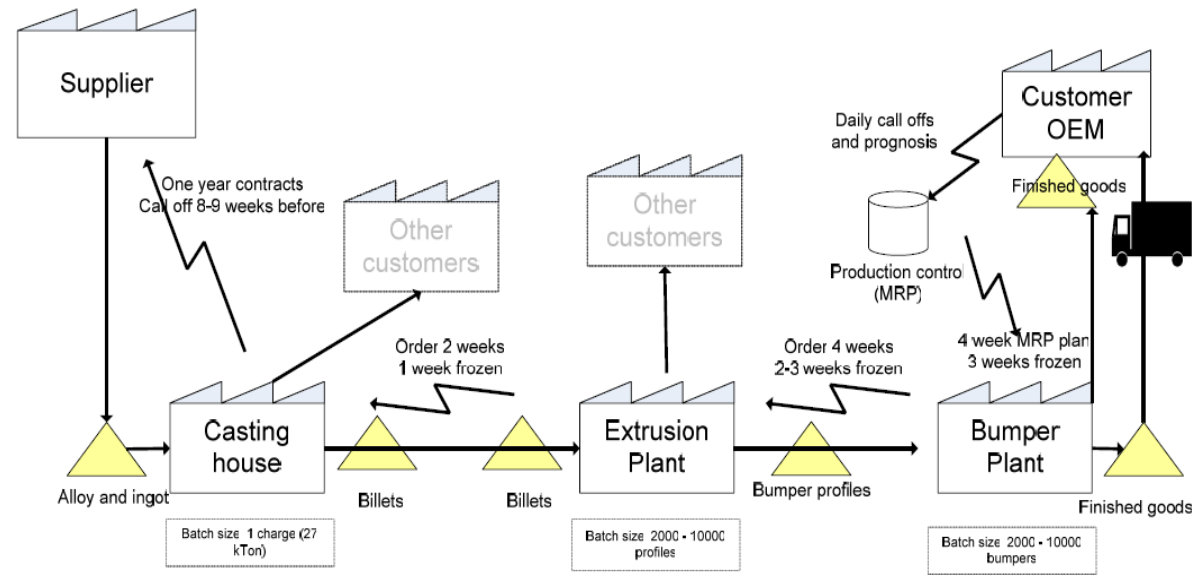

Fig. 2. Current State Map at HARA (Fauske et al., 2008) 
In order to develop an EPE plan for the bumper plant, HARA's products were divided into runners, repeaters and strangers according to demand volume. A range of cost-effective batch sizes were then calculated for the runners. These calculations were based on EOQ calculations, which are quite insensitive across a large interval. That is, EOQ calculations actually indicate a range of batch sizes for which total costs are approximately the same (see Hopp and Spearman, 2000).

The range of cost-effective batch sizes were used together with demand averages to determine how frequently a product should be produced, for example every week or every day, etc. It was established that an EPE plan with weekly cycles could be developed. As far as sequencing was concerned, similar products were placed after each other in order to simplify changeovers between them. This resulted in a fixed cyclic production plan for runners with short lead times and high responsiveness.

For repeaters, time slots were provided in the EPE plan where no production of runners was planned. Lower demand and more variability made repeaters more difficult to introduce into a fixed weekly cyclic schedule. They are therefore produced at a lower frequency, such as every second or even third week, depending on demand and batch size requirement. It was recommended that strangers be carefully investigated as to whether they are actually profitable, considering both the administrative cost and the production cost incurred from making them.

An example of a levelled EPE plan can be seen in Figure 3:

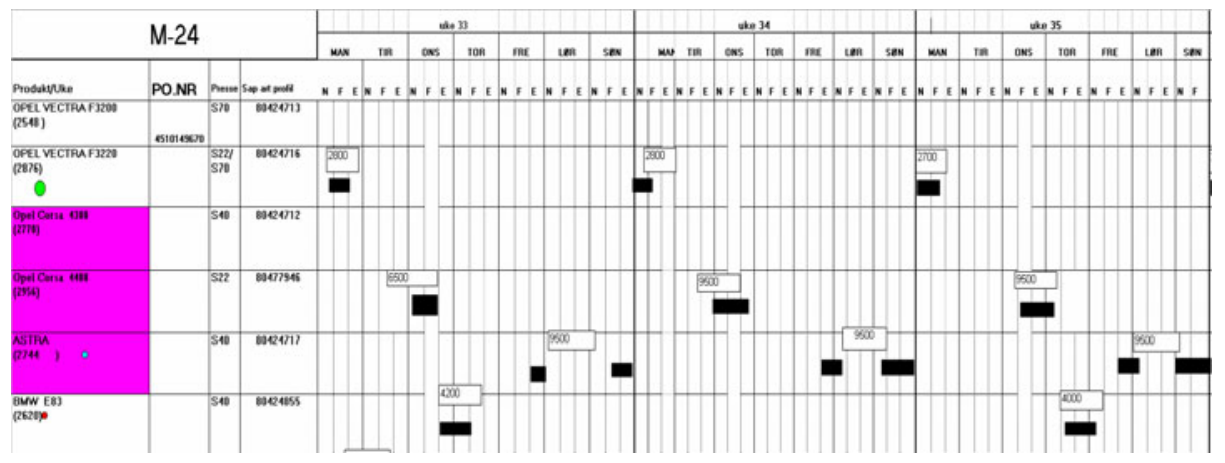

Fig. 3. HARA's EPE Plan

This EPE plan is communicated upstream to improve stability across the entire value-stream. In order to achieve the excess capacity required to fulfil more changeovers and accommodate smaller batches, it was suggested that HARA apply the quick changeover methodology (SMED) to reduce the current setup times. In doing so, lead time and uncontrolled inventory levels will be reduced. Returning back to the original problems of frequent plan changes, the focus on high resource utilisation and large batch sizes, and poor information exchange, the fixed EPE plan allows the smoothing of day-to-day variation by balancing production to longer term demand. Also, EPE 
focuses on batch reduction through continuous setup reduction, and simplifies communication across the entire value-stream.

\section{Conclusion and Further Work}

The traditional lean production control methods of Heijunka and Kanban are difficult to apply in the process-type industries where resource utilisation and large batch runs are the key due to large machines with significant changeover times. On the other hand, the EPE concept appears to have promising results for applying lean production control methods to process-type industries. Based on cyclic planning, the EPE concept is a descendant of the lean movement and introduces a fixed cyclic production plan in order to increase stability and predictability. The positive effects realised through the application of an EPE plan are as a result of this, and include improved coordination across the entire value-stream and the potential for reduced changeover times leading to reduced batch sizes and increased throughput. In the HARA case, it was illustrated how such a plan can improve stability across the entire value-stream.

A key element in realising a fixed EPE plan is the establishment of a finished goods inventory buffer that absorbs fluctuations in demand. Although this may initially increase inventory, it is essential in creating stability on the shopfloor. This stability enables a greater focus on the reduction of changeover times, which in turn allows for the reduction of batch sizes and inventory.

Future work at HARA will include further analysis and synchronisation of the EPE plan across the entire value-stream. We have shown how the EPE concept has potential for application in an automotive, process-type industry. Another area of future research will involve applying the EPE concept to other process-type industries outside of the automotive arena.

\section{References}

Andersen, B., Strandhagen, J.O., Haavardtun, L.J.: Material- og Produksjonsstyring. Cappelen, Oslo

Bicheno, J., Holweg, M.: The Lean Toolbox, 4th edn. PICSIE Books, Buckingham (2009)

Fauske, H., Alfnes, E., Semini, M.: Lean Supply Chain Control in Hydro Automotive Structures. In: APMS 2008 Conference Proceedings (2008)

Glenday, I., Sather, R. (2005), Breaking Through to Flow, http: / / www. leanuk.org/downloads/LFL_2005/Day2_Plenary_Glen day_Sather.pdf (accessed May 2009)

Groenevelt, H., Johansen, J., Lederer, P.J.: Cyclic Planning. University of Rochester, New York (1992)

Hayes, R.H., Wheelwright, S.C.: Link manufacturing process and product life cycles. Harvard Business Review, 133-140 (January-February 1979)

Hill, T.: Operations Management. Palgrave Macmillan, New York (2005) 
Hopp, W., Spearman, M.: Factory Physics. McGraw-Hill, New York (2000)

Inmann, R.R., Jordan, W.C.: Integrated Assembly Line Loading, Design and Labour Planning. Journal of Manufacturing Systems 16(5), 315-322 (1997)

King, P.L.: Lean for the Process Industries. Productivity Press, New York (2009)

Rother, M., Shook, J.: Learning to See. Lean Enterprise Institute Inc, Cambridge (2003)

Shingo, S.: A Study of the Toyota Production System. Productivity Press, New York (1989)

Wild, R.: Production Operations Management. Holt, Rinehart and Winston, New York (1980) 